\title{
The role of Arabidopsis 5PTase13 in root gravitropism through modulation of vesicle trafficking
}

\author{
Yuan Wang ${ }^{1}$, Wen-Hui Lin ${ }^{1}$, Xu Chen ${ }^{1}$, Hong-Wei Xue ${ }^{1}$ \\ ${ }^{I}$ National Key Laboratory of Plant Molecular Genetics, Institute of Plant Physiology and Ecology, Shanghai Institutes for Biologi- \\ cal Sciences, Chinese Academy of Sciences, 300 Fenglin Road, Shanghai 200032, China
}

Inositol polyphosphate 5-phosphatases (5PTases) are enzymes of phosphatidylinositol metabolism that affect various aspects of plant growth and development. Arabidopsis 5PTase13 regulates auxin homeostasis and hormonerelated cotyledon vein development, and here we demonstrate that its knockout mutant 5 pt 13 has elevated sensitivity to gravistimulation in root gravitropic responses. The altered responses of $5 \mathrm{pt13}$ mutants to 1- $\mathrm{N}$-naphthylphthalamic acid (an auxin transport inhibitor) indicate that 5PTase13 might be involved in the regulation of auxin transport. Indeed, the auxin efflux carrier PIN2 is expressed more broadly under 5PTase13 deficiency, and observations of the internalization of the membrane-selective dye FM4-64 reveal altered vesicle trafficking in 5pt13 mutants. Compared with wild-type, 5pt13 mutant seedlings are less sensitive to the inhibition by brefeldin A of vesicle cycling, seedling growth, and the intracellular cycling of the PIN1 and PIN2 proteins. Further, auxin redistribution upon gravitropic stimulation is stimulated under 5PTase13 deficiency. These results suggest that 5PTase13 may modulate auxin transport by regulating vesicle trafficking and thereby play a role in root gravitropism.

Keywords: Arabidopsis, 5PTase13, vesicle trafficking, gravitropism, polar auxin transport

Cell Research (2009) 19:1191-1204. doi: 10.1038/cr.2009.105; published online 8 September 2009

\section{Introduction}

The phosphatidylinositol (PI) signaling pathway participates in multiple processes of plant growth and development and plays a key role in cell responses to a variety of environmental stimuli [1-3]. Inositol polyphosphate 5-phosphatase (5PTase), a key enzyme in the PI pathway, can dephosphorylate the second messenger molecules inositol 1,4,5-trisphosphate $\left(\operatorname{Ins}(1,4,5) \mathrm{P}_{3}\right)$ or inositol 1,3,4,5-tetrakisphosphate $\left(\operatorname{Ins}(1,3,4,5) \mathrm{P}_{4}\right)$ by removing the phosphate group at the 5-position of the inositol ring. Fifteen genes are currently recognized in Arabidopsis thaliana that encode 5PTases [4], and bio-

Correspondence: Hong-Wei Xue

Tel: +86-21-54924059; Fax: +86-21-54924060

E-mail: hwxue@sibs.ac.cn

Abbreviations: PI (Phosphatidylinositol); 5PTases (Inositol polyphosphate 5-phosphatases); Ins (1,4,5) $\mathrm{P}_{3}$ (Inositol 1,4,5-trisphosphate); PAT (Polar auxin transport); BFA (Brefeldin A); PIN (PIN-FORMED); NPA (1- $N$ naphthylphthalamic acid)

Received 10 April 2008; revised 27 August 2008; accepted 2 April 2009; published online 8 September 2009 chemical studies indicate that they can specifically hydrolyze water-soluble inositol phosphates, lipid-soluble phosphoinositides, or both in vitro [4-7].

Functional studies have demonstrated the diverse roles of 5PTases in various developmental processes. COTYLEDON VEIN PATTERN 2 is essential for the closed venation patterns of foliar organs [8], and 5PTase13 modulates cotyledon vein development by regulating auxin homeostasis [9]. Additionally, FRAGILE FIBER 3, which exhibits phosphatase activity toward both inositol phosphate and phosphoinositides, is required for secondary wall synthesis and actin organization in fiber cells [6]. Recently, Gunesekera et al. [10] demonstrated that 5PTase 1 and 5PTase 2 are required for seedling growth. Moreover, it is worthwhile to note that most reported 5PTases are involved in abscisic acid responses [9-12]. As a substrate of 5PTases, Ins $(1,4,5) \mathrm{P}_{3}$ seems to be important in these developmental processes and responses [8-12].

Gravity significantly affects plant growth and development [13]. In response to gravity, plant shoots grow upward (negative gravitropism) and roots downward (positive gravitropism). The process of gravitropic response 
includes sensing gravity direction and subsequently converting it into internal signals. These signals are then transferred to the responding tissues, resulting in organ bending [14]. Gravity perception and the gravitropic response are mediated by a comprehensive signaling network in which auxin and its polar transport play crucial roles [15]. Altered polar auxin transport (PAT) and the consequent redistribution of auxin following gravity stimulation initiate differential growth, which results in the tropistic bending of plants [16].

Several auxin efflux carriers designated as PIN proteins are essential for auxin distribution and root gravitropism. PIN2 localizes to the upper membrane in the epidermis/lateral root cap and preferentially to the lower membrane in cortex cells $[17,18]$, and is required for root gravitropism by regulating auxin basipetal transport from the root tip toward the elongation zone [17-19]. Gravistimulation promotes the expression and polar localization of PIN2 from the root tip to the root elongation zone, and the overexpression of PIN2 results in an enhanced tropistic response [20], indicating that a direct relationship exists between PIN2 expression and the root gravitropic response. Additionally, PIN3, which is responsible for auxin lateral redistribution in root columella upon gravistimulation, is crucial for gravitropism as well [21].

Previous studies showed that PIN1 and PIN2 cycle between the plasma membrane and endosomal compartments in a vesicle trafficking- and actin-dependent manner, and this pattern is of great significance to the polar localization and auxin efflux capacity of PIN proteins [22-24]. PIN1 cycling is dependent upon GNOM ARFGEF and sensitive to the vesicle-trafficking inhibitor brefeldin A (BFA). In addition, the basal PIN2 in cortex cells and apical PIN2 in epidermal cells may have differential recycling machineries dependent on distinct ARFGEF vesicle-trafficking regulators [25]. More recently, it is shown that the vacuolar targeting of PIN2 is dependent on retromer and may be responsible for the differential degradation of PIN2 in gravitropic response [26, 27]. These findings suggest that vesicle trafficking likely mediates the transport of auxin flux carriers to ensure their proper localizations and auxin flux, which in turn modulates auxin-regulated plant growth and development. Additionally, phospholipase D and phosphatidic acid, two important components in the PI signaling pathway, participate in vesicle trafficking in both mammalian and plant cells. They regulate the auxin response by stimulating vesicle trafficking [28-31].

Ins $(1,4,5) \mathrm{P}_{3}$, a putative substrate of 5PTases, plays crucial roles in multiple signaling cascades and plant gravitropic responses. The biphasic change of $\operatorname{Ins}(1,4,5)$
$\mathrm{P}_{3}$ upon gravistimulation occurs in the sensing and responding tissues, but not in the non-elongating internodal regions of stems $[32,33]$. Recent studies revealed that in Arabidopsis inflorescence stems, $\operatorname{Ins}(1,4,5) \mathrm{P}_{3}$ levels increased by two- to three-fold over the first $5 \mathrm{~min}$, followed by a second increase with a peak around 15-20 min after gravistimulation. This correlated well with the initiation of the bending response, indicating the role of $\operatorname{Ins}(1,4,5) \mathrm{P}_{3}$ in the curvature [34]. Indeed, with an impaired Ins $(1,4,5) \mathrm{P}_{3}$ signal, the gravitropic bending of roots, hypocotyls, and inflorescence stems in plants is reduced by approximately $30 \%$ [34].

Although Ins $(1,4,5) \mathrm{P}_{3}$ has immediate relevance to plant gravitropic responses, none of the Arabidopsis 5PTases has been reported to be involved in this process. Here, we show that the 5 pt 13 mutant, which has altered auxin homeostasis and signaling [9], displays an enhanced root gravitropic response and reduced response to the auxin efflux inhibitor 1-N-naphthylphthalamic acid (NPA). A deficiency in 5PTase13 resulted in expanded PIN2 expression in roots and altered vesicle transport. 5 pt13 mutant seedlings were less sensitive to the vesicletrafficking inhibitor BFA and showed reduced BFA effects on PIN2 intracellular cycling, as well as on that of PIN1. As a result of the altered intracellular cycling of PIN proteins, the gravity-induced auxin redistribution was stimulated in the $5 p t 13$ mutant. These results indicate that 5PTase 13 functions in root gravitropism by regulating vesicle trafficking and gravity-induced auxin redistribution.

\section{Results}

5 pt13 mutant seedlings display elevated sensitivity to gravistimulation and are less sensitive to NPA treatment

Previous studies showed that $\operatorname{Ins}(1,4,5) \mathrm{P}_{3}$ is involved in the gravitropic response. Based on the fact that Arabidopsis 5PTases dephosphorylate $\operatorname{Ins}(1,4,5) \mathrm{P}_{3}$ and Ins $(1,3,4,5) \mathrm{P}_{4}$, we tried to test whether Arabidopsis 5PTases participate in plant gravitropism by testing the gravitropic response of the $5 \mathrm{pt} 13$ mutant, which has defective expression of 5PTase13 [9]. Measurement and statistical analysis of the root curvature of $5 \mathrm{pt} 13$ mutant and wild-type (WT) seedlings after gravistimulation $\left(180^{\circ}\right)$ showed an enhanced response in the 5 pt13 mutant. After $4 \mathrm{~h}$ of reorientation, the bending angle of $5 \mathrm{pt} 13$ mutant roots reached $50^{\circ}$, whereas that of WT was only $37^{\circ} .5 p t 13$ mutant seedlings showed a greater curvature than WT within $12 \mathrm{~h}$ after reorientation (Figure $1 \mathrm{~A})$; this indicates an elevated sensitivity of $5 p t 13$ mutant seedlings to gravistimulation (both WT and 5 pt13 mutant seedlings reached $180^{\circ}$ after $48 \mathrm{~h}$ ). Re-expression 
A

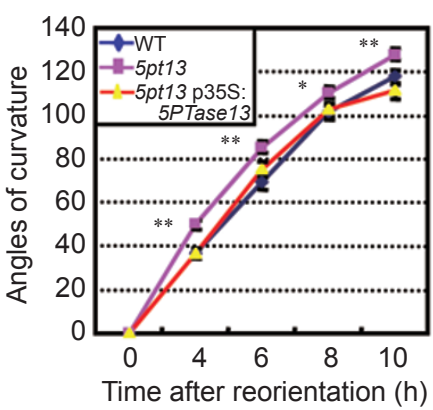

B

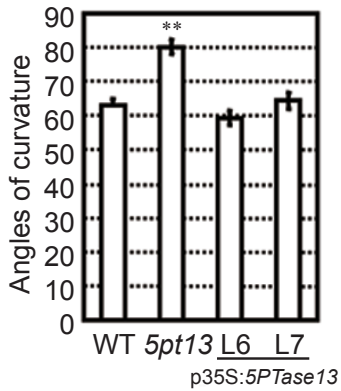

C

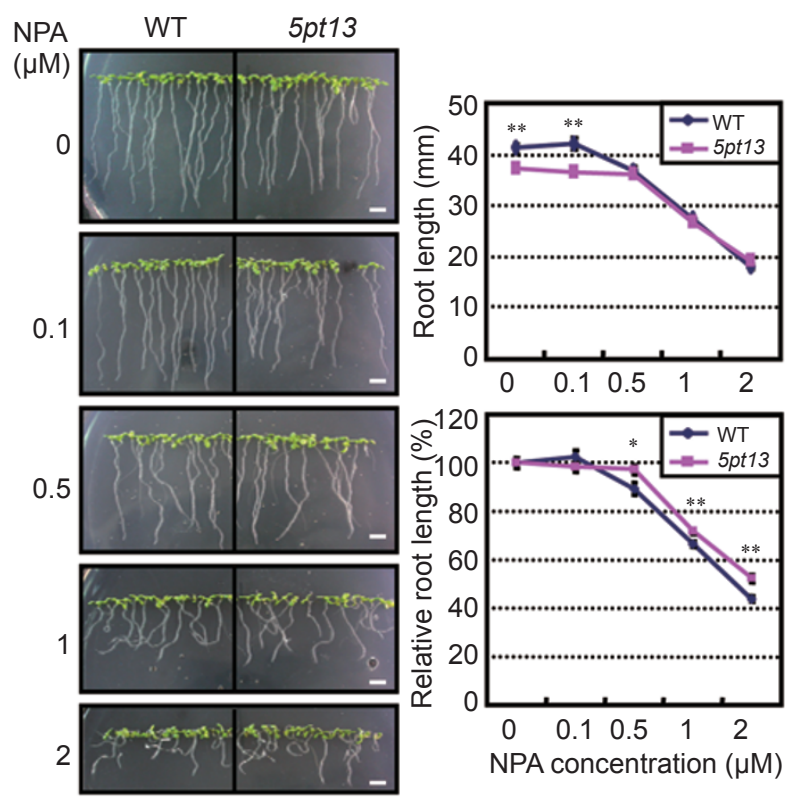

Figure 1 The $5 p t 13$ mutant has an enhanced root gravitropic response and a reduced response to the auxin efflux inhibitor NPA. (A) The $5 p t 13$ mutant displayed elevated sensitivity to gravistimulation. Seedlings (3-day old) were rotated $180^{\circ}$ in the dark, and root tropistic bending curvatures of WT, $5 p t 13$, and 5 pt13 seedlings overexpressing 5PTase13 (p35S:5PTase13 in $5 p t 13$ ) were measured at intervals of $2 \mathrm{~h}$. Statistical analysis using SAS statistical software indicated significant differences $\left({ }^{*} P\right.$ $<0.05$; ** $P 0.01)$. Error bars represent SE $(n=60)$ in A and B. (B) 5PTase13-overexpressing seedlings showed similar gravitropic responses to WT seedlings. The root gravitropic responses of WT, 5pt13, and 5PTase13-overexpressing (p35S:5PTase13 lines L6, L7) seedlings were measured $6 \mathrm{~h}$ after reorientation. (C) The root growth of the $5 p t 13$ mutant was less sensitive to the auxin efflux inhibitor NPA. WT and $5 p t 13$ mutant seedlings were vertically grown for 8 days in the absence or presence of NPA $(0.1,0.5,1$, or $2 \mu \mathrm{M}$, left panel, Bar $=5 \mathrm{~mm})$, followed by measurement of the lengths of primary roots (right-top panel) and calculation of relative root lengths (right bottom panel). The root lengths of untreated WT and $5 p t 13$ mutant seedlings were set to $100 \%$. Statistical analysis using SAS statistical software indicated significant differences $(* P<0.05 ; * * P<0.01)$. Error bars represent SE $(n=50)$. of the 5PTase 13 gene in the 5 pt13 mutant (by introducing p35S:5PTase 13 in 5pt13 lines) rescued the enhanced gravity response, which confirms that the stimulated gravitropic response in the $5 p t 13$ mutant was due to the disruption of the 5PTase13 gene. However, overexpression of 5PTase 13 had no effect on the gravitropic response (Figure 1B, p35S:5PTase13 lines 6, 7).

Based on the significant role of PAT in plant gravitropism, the enhanced gravitropic response of $5 \mathrm{pt} 13$ mutant seedlings raised the possibility that PAT might be modulated. Indeed, analysis of the responses of WT or 5 pt13 mutant seedlings to NPA, an auxin efflux inhibitor, revealed a reduced inhibitory effect of NPA in $5 p t 13$ mutant seedlings. NPA $(0.5 \mu \mathrm{M})$ treatment suppressed the root growth of WT seedlings by $11 \%$, but had little effect on the root growth of 5 pt13 seedlings (Figure 1C). Following treatment with exogenous 1 or $2 \mu \mathrm{M} \mathrm{NPA}$, the root lengths of WT seedlings were 67 and $44 \%$ of untreated controls, whereas those of $5 p t 13$ seedlings were 72 and $52 \%$, respectively (statistically significantly different from WT). This observation indicates an attenuated effect of NPA on 5 pt13 mutant seedlings, and suggests that the enhanced gravitropic response of the 5 pt13 mutant may be attributed to the stimulated PAT, especially auxin efflux.

\section{PTase13 deficiency results in expanded expression of} PIN2 proteins in roots

Muller et al. [17] demonstrated that PIN2 is required for root gravitropism through the regulation of auxin distribution from the root tip toward the elongation zone. We further explored the effect of 5PTase13 on PIN2 by introducing a fused PIN2-EGFP (enhanced green fluorescent protein) [35] expression cassette into the $5 p t 13 \mathrm{mu}-$ tant via genetic crosses. Examination of the expression and polar localization of PIN2 in the homozygous cross progenies (PIN2-EGFP/5pt13) showed that there was no change in PIN2 polar localization under 5PTase13 deficiency in the 5 pt 13 mutant; however, the region of PIN2 expression was greatly expanded from the root meristem zone to the elongation zone. Detailed measurements showed that compared with that of PIN2-EGFP seedlings $(424.01 \pm 16.3 \mu \mathrm{m})$, the PIN2 expression region (from the root tip) was much broader in PIN2-EGFP/5pt13 roots $(529.70 \pm 17.8 \mu \mathrm{m}$ ), with an increase of $\sim 24 \%$ (Figure $2 \mathrm{~A})$. This is in agreement with a previous report that the tropistic response is enhanced in seedlings overexpressing PIN2 [20]; and the broader PIN2 expression of 5 pt 13 mutant seedlings may contribute to the stimulated gravitropic response.

Further support came from observations following application of exogenous $\operatorname{Ins}(1,4,5) \mathrm{P}_{3}(10 \mu \mathrm{M})$, the 
substrate of $5 \mathrm{PT}$ Tase 13 , to WT seedlings. The intrinsic Ins $(1,4,5) \mathrm{P}_{3}$ is indeed increased by using an intracellular delivery system (Supplementary information, Figure S1), which expanded the PIN2 expression region (Figure 2B), while application of Ins $(1,3,4) \mathrm{P}_{3}$ (inositol 1,3,4-trisphosphate, $10 \mu \mathrm{M}$ ) had no effect. This finding was consistent with the broader expression of PIN2 in 5 pt13 mutant seedlings. To test whether the effect of $\operatorname{Ins}(1,4,5) \mathrm{P}_{3}$ requires de novo protein synthesis, a protein synthesis inhibitor, cycloheximide (CHX), was used. Results show that the PIN2 expression region was similarly expanded upon $\operatorname{Ins}(1,4,5) \mathrm{P}_{3}$ treatment in the presence of $\mathrm{CHX}$ (Figure 2B). Further examination of transcription of PIN2 using PIN2p::GUS seedlings (kindly provided by Dr Jian $\mathrm{Xu}$ ) shows that no significant difference in GUS activity was detected after Ins $(1,4,5) \mathrm{P}_{3}$ treatment (Figure $2 \mathrm{C}$, left panel), confirming that $\operatorname{Ins}(1,4,5) \mathrm{P}_{3}$ had no effect on the transcription of PIN2. Quantitative analysis of PIN2 transcripts in root tissues also confirmed that the transcription of PIN2 in 5 pt13 root tissues was similar to that in WT roots (Figure 2C, right panel). Together, these findings indicate potential post-transcriptional regulation of PIN2.

5PTase13 deficiency results in altered vesicle trafficking and the 5 pt 13 mutant is less sensitive to BFA inhibition

PIN1 and PIN2 proteins are cycled between the plasma membrane and endosomal compartments, and vesicle trafficking is crucial for this process [22-25]. To investigate the mechanism of expanded PIN2 expression, the membrane-selective fluorescent dye FM4-64, a widely used tracer for endocytic trafficking [36], was employed to monitor vesicle transport behavior in root cells. Our observations showed that the internalization of FM4-64 was significantly enhanced in 5 pt 13 mutant roots (more vesicles labeled by FM4-64, Figure 3A).

BFA, a well-known vesicle-trafficking inhibitor, specifically blocks exocytosis (but not endocytosis) and promotes vesicle aggregation to form BFA compartments [37]. BFA can also mimic the physiological effects of auxin transport inhibitors on root and hypocotyl elongation, and lateral root development [22]. Based on the enhanced internalization of FM4-64 under 5PTase13 deficiency, BFA compartment formation in the 5 pt13 mutant was further examined. The results showed that BFA treatment did not block the cycling of endocytic vesicles entirely in 5 pt 13 root cells. Moreover, smaller BFA compartments were formed (Figure 3B), which suggests a decreased sensitivity of vesicle transport to BFA in the 5pt13 mutant. Combined with the enhanced internalization of FM4-64, these findings indicate altered vesicle trafficking in 5 pt13 mutant seedlings. Further
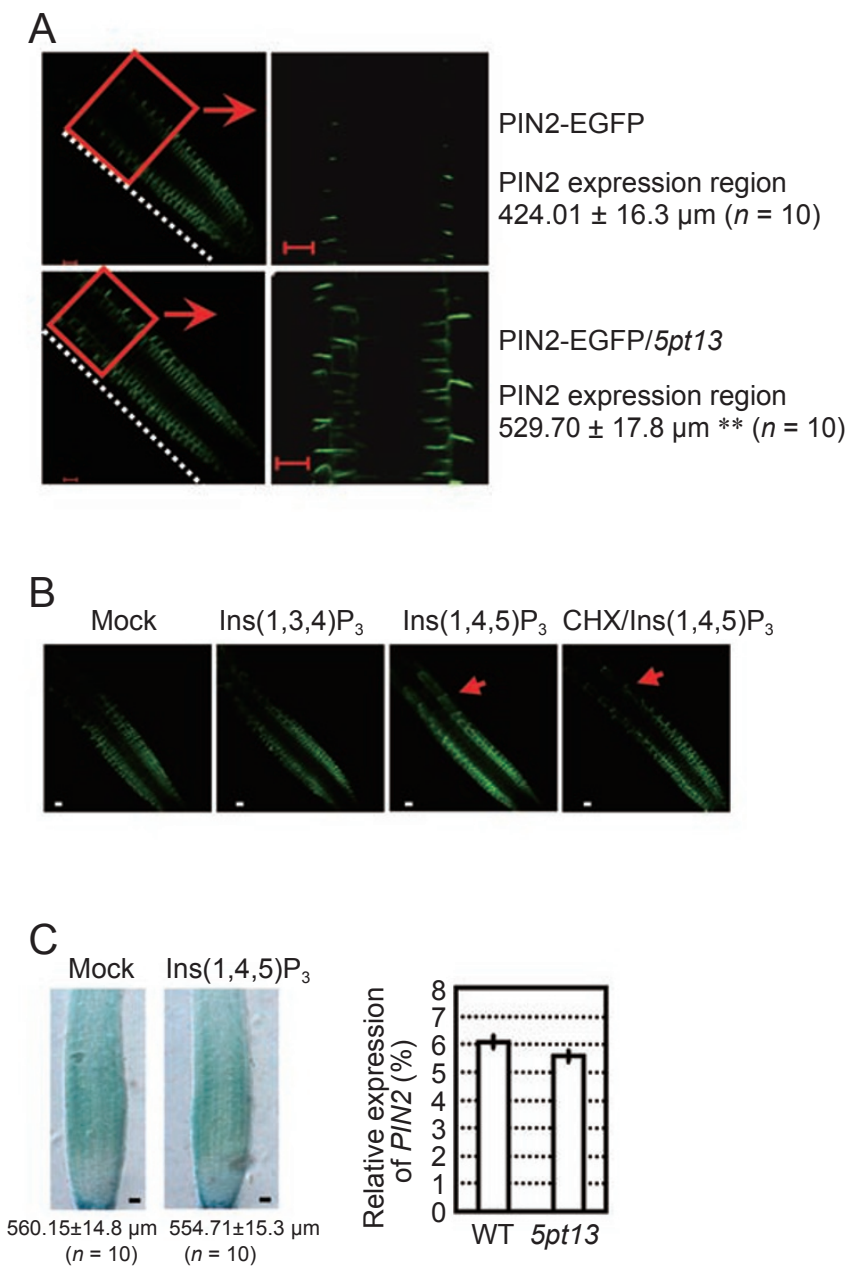

Figure 2 Expanded PIN2 expression under 5PTase13 deficiency or exogenous Ins $(1,4,5) P_{3}$. (A) PIN2 expression in PIN2EGFP/5pt13 (panel 2) was expanded to root elongation zones by $\sim 24 \%$ relative to PIN2-EGFP controls. Discontinuous lines represent the PIN2 expression domains (from the root tip). Statistical analysis using SAS statistical software indicated a significant difference $(* * P<0.01)$. Data are presented as means \pm SE $(n$ $=10)$. Bar $=20 \mu \mathrm{m}$, same in B and C. (B) Exogenous Ins $(1,4,5) \mathrm{P}_{3}$ (Inositol 1,4,5-trisphosphate) stimulated PIN2 expression in the root elongation zones. WT seedlings (4-day-old) were treated with $10 \mu \mathrm{M}$ Ins $(1,3,4) \mathrm{P}_{3}$ (Inositol 1,3,4-trisphosphate), Ins $(1,4,5) \mathrm{P}_{3}$, or a control solution for $2 \mathrm{~h}$ before observation. Seedlings pretreated with $50 \mu \mathrm{M}$ cycloheximide ( $\mathrm{CHX}$ ) for $30 \mathrm{~min}$ were incubated with $\operatorname{Ins}(1,4,5) \mathrm{P}_{3}$ for $2 \mathrm{~h}$ in the presence of $\mathrm{CHX}$. A total of 20 seedlings were observed after each treatment. (C) $\operatorname{lns}(1,4,5) P_{3}$ had no effect on transcriptional expression of PIN2 gene. PIN2p::GUS lines were treated with $\operatorname{Ins}(1,4,5) \mathrm{P}_{3}$ as mentioned in $(B)$ and transcriptional region of $P I N 2$ gene was measured and analyzed (left panel). Quantitative real-time RT-PCR analysis revealed that there was no distinct difference in PIN2 expression levels between WT and 5 pt13 roots. The relative expression levels of the PIN2 gene, normalized against that of the ACTIN7 gene, are presented (percentages, right panel). Data are from three independent experiments, and error bars indicate SE. Statistical analysis indicated no significant difference. 
Table 1 Quantitative analyses of intracellular vesicle cycling

\begin{tabular}{|c|c|c|c|c|c|c|}
\hline & \multicolumn{4}{|c|}{ FM4-64 staining } & \multicolumn{2}{|c|}{ BFA treatment } \\
\hline & \multirow[b]{2}{*}{ WT } & & \multicolumn{2}{|c|}{ p35S:5PTase13 } & \multirow[b]{2}{*}{ WT } & \multirow[b]{2}{*}{$5 p t 13$} \\
\hline & & $5 p t 13$ & L6 & L7 & & \\
\hline$P$ value & & $9.10 \mathrm{E}-61$ & 0.12 & 0.89 & & $1.36 \mathrm{E}-37$ \\
\hline
\end{tabular}

The observation and measurement performed were with the help of confocal laser scanning microscope. For each seedling, the vesicle numbers are from the average of 10 root cells. The data are presented as mean $\pm \mathrm{SE}$ ( $n=30$ individual seedlings). $P$-values were statistically calculated with SAS software.

A

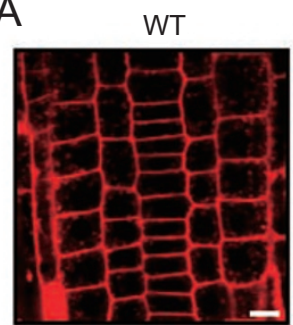

B

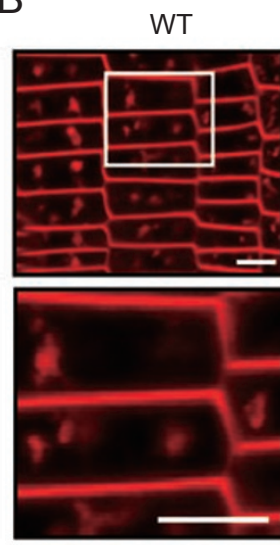

C

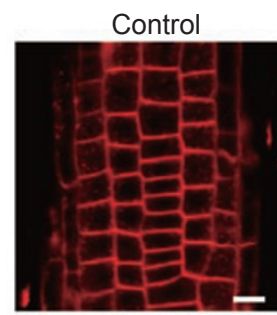

$5 p t 13$

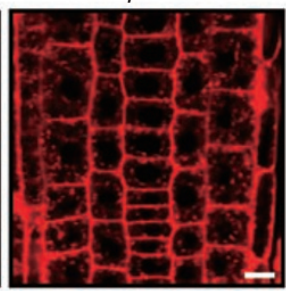

$5 p t 13$

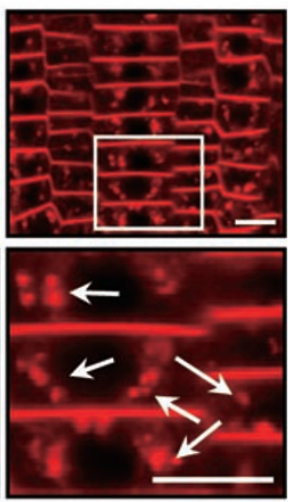

D
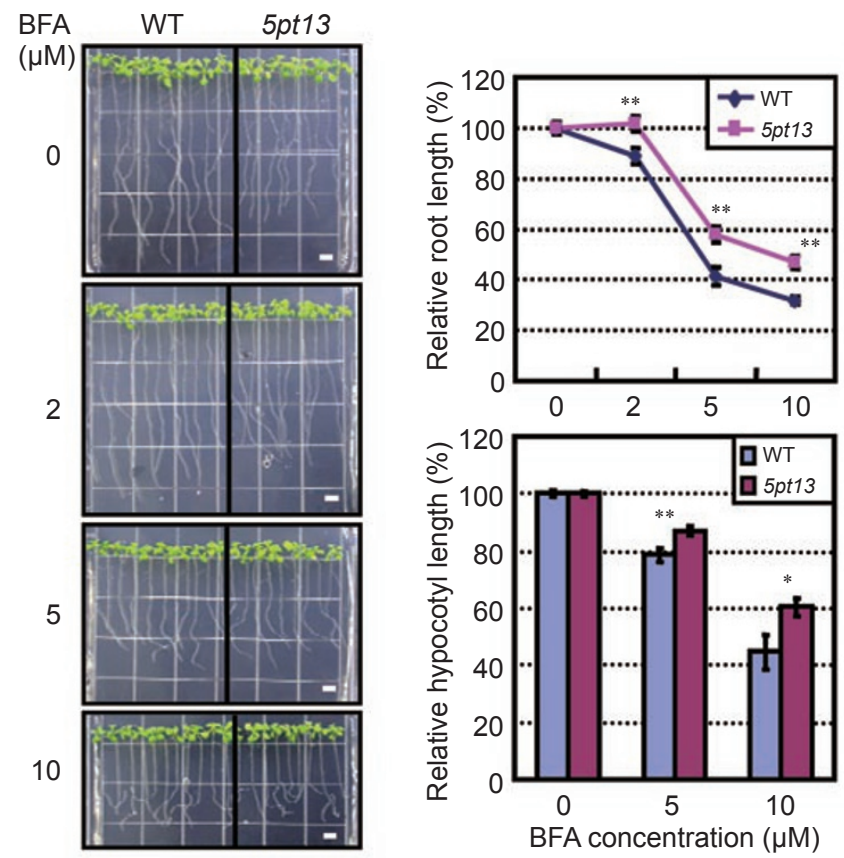

Figure 3 Altered vesicle trafficking under 5PTase13 deficiency. (A) Internalization of FM4-64 in root cells of the $5 p t 13$ mutant was stimulated relative to that of WT. Vesicles were stained with FM 4-64 for $40 \mathrm{~min}$. Bar = $10 \mu \mathrm{m}$. Images in A, B, and C are representative of 30 individual samples. (B) Vesicle trafficking in 5 pt13 mutant seedlings was less inhibited by BFA relative to that in WT seedlings. The squared regions are enlarged to highlight the differences. The internalization of FM4-64 was observed after treatment with BFA $(100 \mu \mathrm{M})$ and FM4-64 $(5 \mu \mathrm{M})$ for $30 \mathrm{~min}$. Bar $=10 \mu \mathrm{m}$. (C) 5PTase13-overexpressing lines (L6, L7) presented normal vesicular trafficking. Bar $=10 \mu \mathrm{m}$. (D) The inhibitory effects of BFA on seedling growth were suppressed under 5PTase13 deficiency. Seedlings (6-day old) were transferred onto medium supplemented with different concentrations of BFA and grown vertically for another 6 days (left panel, Bar $=5 \mathrm{~mm}$ ). The relative lengths of roots (right upper panel) and hypocotyls (grown in the dark, right bottom panel) were calculated. The lengths of the primary roots and hypocotyls of untreated plants were set to $100 \%$. Statistical analysis using SAS statistical software indicated significant differences $(* P$ $<0.05 ; * * P<0.01)$. Error bars represent SE $(n=50)$. 
quantitative analyses revealed similar results (Table 1), consistent with these observations. In contrast, FM4-64 internalization in 5PTase 13-overexpressing seedlings (lines 6,7) was not evidently altered (Figure 3C), which indicates normal vesicle trafficking and is consistent with their indistinguishable gravitropic response under gravistimulation.

Further studies on physiological responses confirmed the resistance of 5 pt 13 mutant seedlings to BFA treatment. In $5 p t 13$ mutant seedlings, the inhibitory effects of BFA on root and hypocotyl growth were not as effective as that in WT (Figure 3D). With increased BFA concentrations (from 0 to $10 \mu \mathrm{M}$ ), the root growth of WT seedlings was gradually inhibited, whereas 5 pt 13 mutant seedlings displayed a relatively mild inhibition. Compared with WT, the inhibitory effects of 2,5 , or $10 \mu \mathrm{M}$ BFA on root growth were suppressed by approximately $15 \%$ in $5 p t 13$ mutant seedlings. Similar results were observed for hypocotyls. Application of $5 \mu \mathrm{M}$ BFA caused a $21.4 \%$ suppression of hypocotyl length in WT, but only a $12.9 \%$ suppression in 5 pt 13 mutant seedlings. Under $10 \mu \mathrm{M}$ BFA treatment, the hypocotyl length of WT decreased to $44.7 \%$ of untreated controls, whereas that of 5 pt 13 mutant was still at $60.4 \%$. The reduced responses to BFA suggest the presence of alternative BFA-resistant intracellular sorting events in the $5 p t 13$ mutant.
A
WT
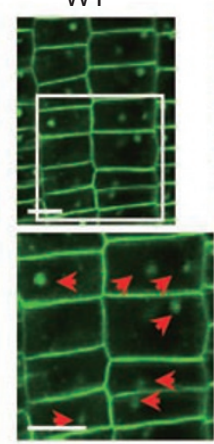

C
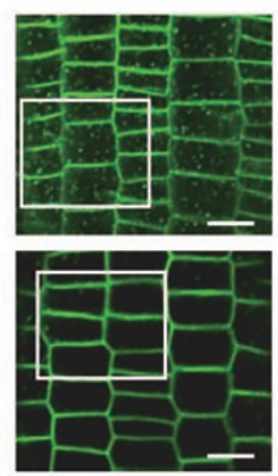

$5 p t 13$
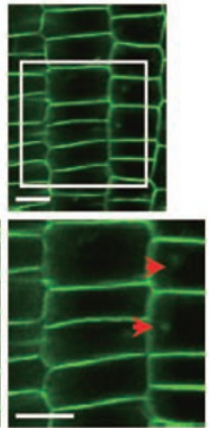

(1)

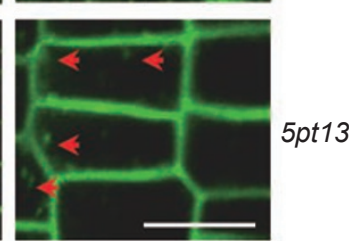

B

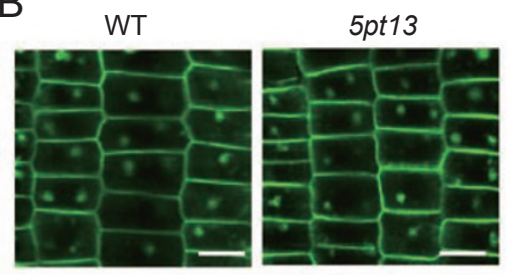

D
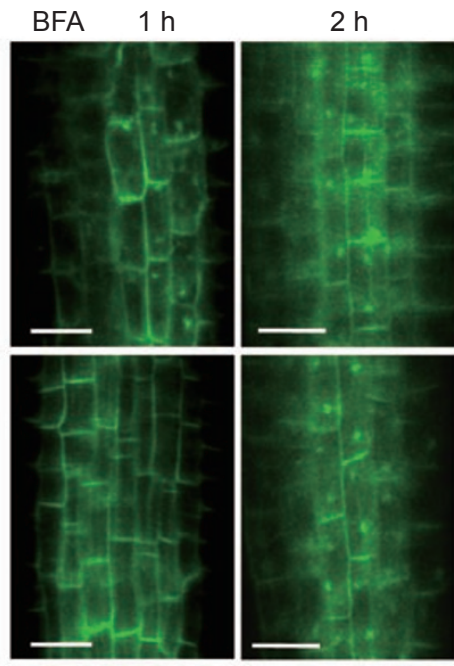

Washout

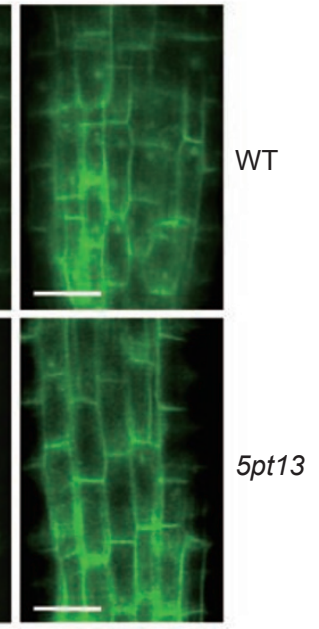

Figure 4 Altered intracellular cycling of PIN2 and PIN1 under 5PTase13 deficiency. (A) Compared with PIN2-EGFP, PIN2EGFP/5pt13 seedlings had fewer BFA compartments (arrows) under BFA treatment $(50 \mu \mathrm{M})$ for $1 \mathrm{~h}$. Bar $=10 \mu \mathrm{m}$. For each treatment in A, B, and C, 30 seedlings were observed. (B) BFA treatment (50 $\mu \mathrm{M})$ for $2 \mathrm{~h}$ resulted in similar PIN2 internalization in cortex cells of both PIN2-EGFP and PIN2-EGFP/5pt13. Bar = $10 \mu \mathrm{m}$. (C) After BFA washout (pretreated with BFA for $2 \mathrm{~h}$ ), a large amount of PIN2-containing vesicles were still observed (arrows) in PIN2-EGFP cortex cells, whereas those in PIN2EGFP/5pt13 cortex cells were hardly detected, revealing enhanced recycling of basal-targeted PIN2 to plasma membrane under 5PTase13 deficiency. Pretreated seedlings (50 $\mu \mathrm{M} \mathrm{BFA}$, for $2 \mathrm{~h})$ were subjected to a 2-h washing out. Bar $=10 \mu \mathrm{m}$. (D) Compared with PIN1-EYFP, the formation of BFA compartments was significantly suppressed in PIN1-EYFP/5pt13 seedlings after BFA treatment for $1 \mathrm{~h}$ (left panel). BFA treatment for $2 \mathrm{~h}$ leads to similar internalization of PIN1 proteins in both PIN1EYFP and PIN1-EYFP/5pt13 seedlings (middle panel). The polar localization of PIN1 could be more rapidly restored in PIN1EYFP/5pt13 seedlings after BFA washout for $1 \mathrm{~h}$ (pretreated with BFA for $2 \mathrm{~h}$ ), whereas PIN1-containing BFA compartments were still observed in PIN1-EYFP seedlings (right panel). This indicates an enhanced recycling of PIN1 to plasma membrane under 5 PTase 13 deficiency. Bar $=10 \mu \mathrm{m}$. For each treatment above, 20 seedlings were observed. 
Reduced effects of BFA on intracellular cycling of PIN1 and PIN2 in the 5pt13 mutant

The cycling of PIN1 and PIN2 is dependent on vesicle transport and they can be internalized by BFA to accumulate in BFA compartments [22-25, 38]. The altered vesicle trafficking in 5 pt13 mutant suggests a potential influence on the intracellular cycling of PIN proteins in cells. In WT seedlings, BFA broke the cycling balance of PIN2 between the plasma membrane and endosomal compartments, and BFA compartments were formed after BFA treatment $(50 \mu \mathrm{M})$ for $1 \mathrm{~h}$, whereas they were much less apparent in PIN2-EGFP/5pt13 seedlings (Figure 4A), indicating that the intracellular cycling of PIN2 proteins was less sensitive to BFA in 5pt13 mutant seedlings.

When BFA treatment lasted for $2 \mathrm{~h}$, the recycling of PIN2 to the plasma membrane was completely inhibited, and most PIN2 proteins aggregated in large BFA compartments in both PIN2-EGFP and PIN2-EGFP/5pt13 seedlings (Figure 4B). Statistical analyses on the total size and intensity of BFA compartments in each cortex cell revealed the very similar PIN2 internalization in PIN2-EGFP and PIN2-EGFP/5pt13 seedlings (Table 2). When BFA was washed out after BFA treatment for $2 \mathrm{~h}$, the resumption of PIN2 polar localization was found to be quicker and more robust in 5 pt13 mutant seedlings (Figure 4C). This indicates that the recycling of basal- targeted PIN2 to the plasma membrane is accelerated under 5PTase 13 deficiency. Statistical analyses of PIN2containing BFA compartments and PIN2-containing vesicles provided further support for stimulated PIN2 recycling in 5 pt 13 mutant seedlings (Table 2).

PIN1, another plasma membrane protein, displayed responses to BFA similar to PIN2 in 5pt13 mutant seedlings (Figure 4D and Table 3), which further suggests alternative BFA-resistant sorting events in the $5 p t 13$ mutant. Under 5PTase 13 deficiency, the reduced BFA inhibition of the cycling of PIN1 and PIN2 proteins, as well as the accelerated recycling of basal-targeted PIN proteins to plasma membrane, suggests a negative effect of 5PTase 13 on the cycling of PIN proteins and thus a negative influence on auxin transport.

\section{Stimulated auxin redistribution upon gravistimulation under 5PTase 13 deficiency}

Both the modulated expression of PIN2 and the modified intracellular cycling of PIN proteins under 5 PTase 13 deficiency could influence auxin distribution, especially upon gravistimulation. To test this, an Arabidopsis DR5GUS reporter line containing a cassette of the GUScoding sequence under the control of a synthetic auxininducible promoter [39] was crossed with the $5 p t 13$ mutant to investigate auxin accumulation and distribution. Analysis of the homozygous cross progenies of

Table 2 Quantitative analyses of PIN2 cycling

\begin{tabular}{|c|c|c|c|c|c|}
\hline & \multicolumn{2}{|c|}{ Cells with BFA compartments (\%) } & & \multicolumn{2}{|c|}{ Cells with PIN2-containing vesicles (\%) } \\
\hline & $\overline{\mathrm{WT}}$ & $5 p t 13$ & & WT & $5 p t 13$ \\
\hline $\operatorname{BFA}(1 \mathrm{~h})$ & $82.7 \pm 2.4$ & $8.9 \pm 1.0^{*}$ & BFA washout & $95.8 \pm 2.0$ & $10.4 \pm 1.2 *$ \\
\hline
\end{tabular}

Observation and measurement were performed with the help of confocal laser scanning microscope. The data are presented as mean \pm SE $(n=30$ individual seedlings). The total fluorescent intensity and size of BFA compartments in each cortex cell were shown in brackets (before BFA washout), which were measured by laser confocal microscope LSM 510 (ver3.2). Statistical analysis by using SAS software indicated the significant differences $(* P<0.01)$.

Table 3 Quantitative analyses of PIN1 cycling

\begin{tabular}{lll}
\hline & \multicolumn{2}{c}{ Cells with BFA compartments (\%) } \\
\cline { 2 - 3 } & WT & $5 p t 13$ \\
\hline BFA (1 h) & $60.8 \pm 1.9$ & $5.4 \pm 0.9 *$ \\
BFA (2 h) & $86.6 \pm 1.0$ & $84.3 \pm 1.1$ \\
& $\left(261.7 \pm 4.5,3.1 \pm 0.1 \mu \mathrm{m}^{2}\right)$ & $\left(256.5 \pm 4.2,3.2 \pm 0.1 \mu \mathrm{m}^{2}\right)$ \\
\hline
\end{tabular}

Observation and measurement ( $n=30$ individual seedlings) were performed as described in Table 2. The total fluorescent intensity and size of BFA compartments in each cell were shown in brackets (before BFA washout). Statistical analysis by using SAS software indicated the significant differences $(* P<0.01)$. 


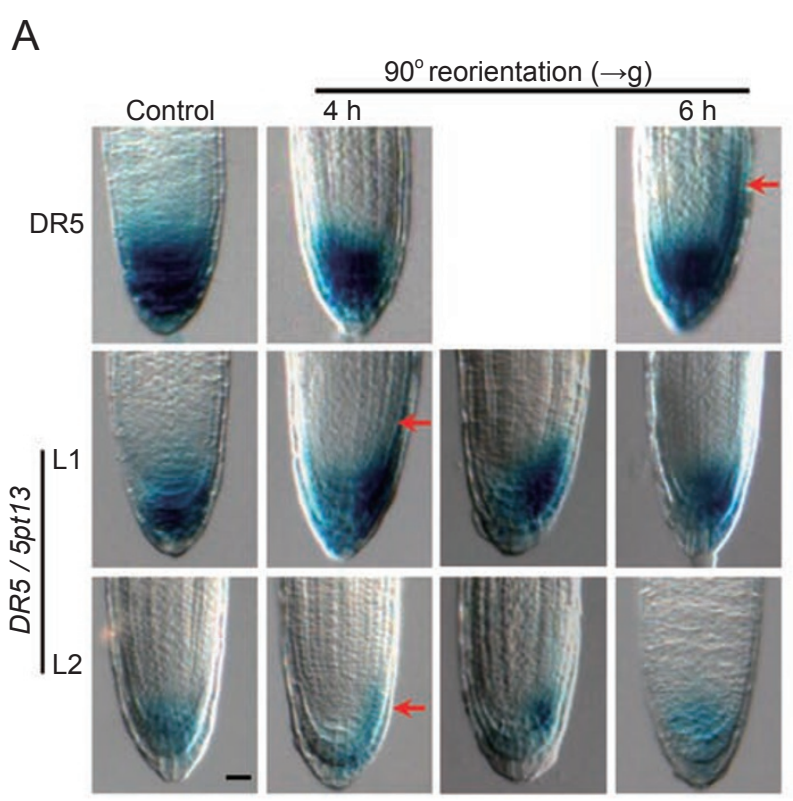

B

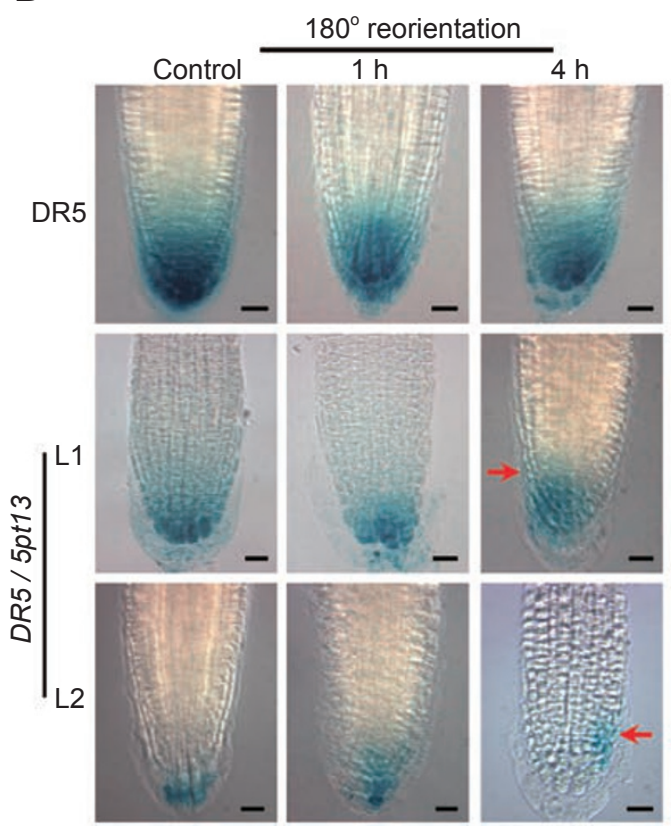

Figure 5 Stimulated auxin redistribution upon gravistimulation under 5PTase13 deficiency. DR5/5pt13 seedlings showed accelerated auxin redistribution under $90^{\circ}(\mathrm{A})$ or $180^{\circ}(\mathrm{B})$ reorientation. The lateral linear GUS activity in a majority of DR5 seedlings was not observed at $4 \mathrm{~h}$ after reorientation under both conditions and was detected after $6 \mathrm{~h}$ of $90^{\circ}$ reorientation, whereas the redistribution of GUS signals could be observed after only $4 \mathrm{~h}$ of reorientation in most DR5/5pt13 roots. Two independent lines, L1 and 2, were observed, and 30 seedlings from each line were used for each treatment. Arrows indicate the streak of lateral GUS activity. Bar $=20 \mu \mathrm{m}$.
DR5/5pt13 showed that auxin in root tips was in an even and symmetric pattern when growing vertically, although the signal was relatively weaker than in DR5 lines owing to decreased auxin content [9]. After a $90^{\circ}$ reorientation for $4 \mathrm{~h}$, the majority of DR5 seedlings still maintained an even and symmetric distribution of auxin in root tips. Statistical analysis indicated that only $\sim 32 \%$ of DR5 seedlings displayed asymmetric auxin distribution, but $\sim 67 \%$ of DR5/5pt 13 seedlings did so (Figure $5 \mathrm{~A}$ ), revealing a significant difference $(P=0.0005)$. The asymmetric auxin in DR $5 / 5$ pt 13 roots was present as a streak in the lower side extending from the tip to the elongation zone (recognized as lateral auxin distribution) or an uneven distribution in root tips (both situations are shown in Figure 5A). In accordance with previous studies [34, 40 ], $\sim 60 \%$ of DR5 seedlings displayed lateral auxin redistribution after $6 \mathrm{~h}$ of reorientation, a time point when DR5/5pt13 seedlings recovered to $\sim 37 \%(P=0.0017)$, indicating a significantly stimulated auxin redistribution under 5PTase13 deficiency. Because lateral auxin distribution was only detected in bending roots with curvatures between $40^{\circ}$ and $50^{\circ}$, and the frequency of asymmetric auxin distribution was tightly correlated with the gravitropic response, the enhanced auxin redistribution in 5PTase 13 mutant indicates the involvement of 5PTase 13 in gravity-induced auxin redistribution.

Similar results were observed when seedlings were reoriented $180^{\circ}$. As shown in Figure 5B, in comparison with DR5 lines, the 5PTase13 deficiency resulted in distinct asymmetric auxin distribution in root tips after a 1 $\mathrm{h}$ reorientation of $180^{\circ}$, which became more distinguishable after $4 \mathrm{~h}$. These results suggest that the stimulated auxin redistribution upon gravistimulation in $5 p t 13 \mathrm{mu}-$ tant was responsible for the enhanced root gravitropic response.

\section{Discussion}

5PTase, a key enzyme in the PI pathway, has been implicated in plant growth and development, as well as responses to environmental stimuli. In this study, we demonstrate the involvement of 5PTase13 in root gravitropism by modulating vesicle trafficking and PAT. Our data provide insight into the role of the PI pathway in the root gravitropic response.

The underlying mechanism of 5PTase 13 in the root gravitropic response

Analysis of the root gravitropic response revealed the enhanced sensitivity of the 5 pt13 mutant to gravistimulation. Although some studies show that $\operatorname{Ins}(1,4,5) \mathrm{P}_{3}$ participates in gravitropic signaling, the involvement 
of Arabidopsis 5PTases in gravitropism has not been previously documented. As a substrate of 5PTase13, previous studies have implicated $\operatorname{Ins}(1,4,5) \mathrm{P}_{3}$ as a signal component in the plant gravitropic response [32, 33], and decreased basal Ins $(1,4,5) \mathrm{P}_{3}$ levels in Arabidopsis result in suppressed gravitropism [34]. These studies suggest a fundamental and extensive role for $\operatorname{Ins}(1,4,5) \mathrm{P}_{3}$ in gravity signaling. Our previous study confirmed the increased Ins $(1,4,5) \mathrm{P}_{3}$ content in 5pt13 mutant seedlings [41] and, indeed, exogenously applied $\operatorname{Ins}(1,4,5) \mathrm{P}_{3}$ promoted gravitropic responses in WT seedlings (Figure 6A), as well in pin 2 mutants (Figure 6B), providing an explanation for the stimulated gravitropism under 5PTase13 deficiency.

Transgenic lines overexpressing 5PTase 13 responded normally to gravistimulation, which can be attributed to an insignificant alteration in $\operatorname{Ins}(1,4,5) \mathrm{P}_{3}$ levels. Previous studies have shown that an extreme decrease (by more than 95\%) in cytosolic $\operatorname{Ins}(1,4,5) \mathrm{P}_{3}$ levels leads to impaired gravitropic bending (of around $20^{\circ}$ ) [34]. This extreme decrease was produced by the overexpression of Hs5PTase, which can specifically hydrolyze $\operatorname{Ins}(1,4,5) \mathrm{P}_{3}$ with high affinity. Although the expression of 5PTase13 gene was indeed enhanced in transgenic lines, the alteration of intrinsic Ins $(1,4,5) \mathrm{P}_{3}$ levels was not as dramatic as that in Hs5PTase-overexpressing lines due to the differential activity and substrate specificity of the enzymes.

Further analysis showed that there was no difference in the agravitropic response of hypocotyls between WT and 5 pt 13 mutants. The fact that almost all Arabidopsis 5PTases (13 of 15 total isoforms) are expressed in the hypocotyl (https://www.genevestigator.ethz.ch/gv/index.jsp) suggests the possible functional redundancy of 5PTases in the hypocotyl.

$\mathrm{Ca}^{2+}$ is regarded as a critical factor in gravitropic signaling and responses, and a global $\mathrm{Ca}^{2+}$ signal is detected in Arabidopsis seedlings under gravistimulation [42]. Given that cytosolic $\mathrm{Ca}^{2+}$ is increased in roots of 5 pt13 seedlings [41], we cannot exclude the possibility that $\mathrm{Ca}^{2+}$ is involved in the enhanced gravitropic response in 5 pt13 mutant seedlings.

Vesicular trafficking-dependent post-Golgi transport has been implicated in shoot gravitropism. Several genes have been identified by defective shoot gravitropic response and found to participate in vacuolar formation, which relies on post-Golgi transport. SHOOT GRAVITROPISM3 and SHOOT GRAVITROPISM4 encode homologs of SNARE proteins and function in vesicular transport to the vacuole $[43,44]$. SGR8, whose animal homolog RME-8 is involved in receptor-mediated endocytosis, may play a role in endocytosis as well $[45,46]$. However, whether vesicle cycling directly participates in the root gravity response is still unknown. Our results
A
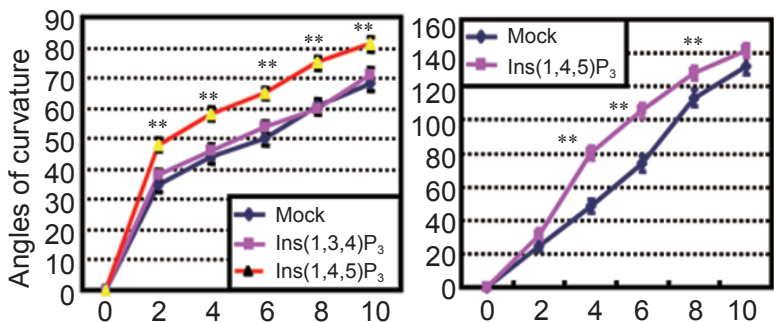

Time after $90^{\circ}$ reorientation (h) Time after $180^{\circ}$ reorientation (h)

B
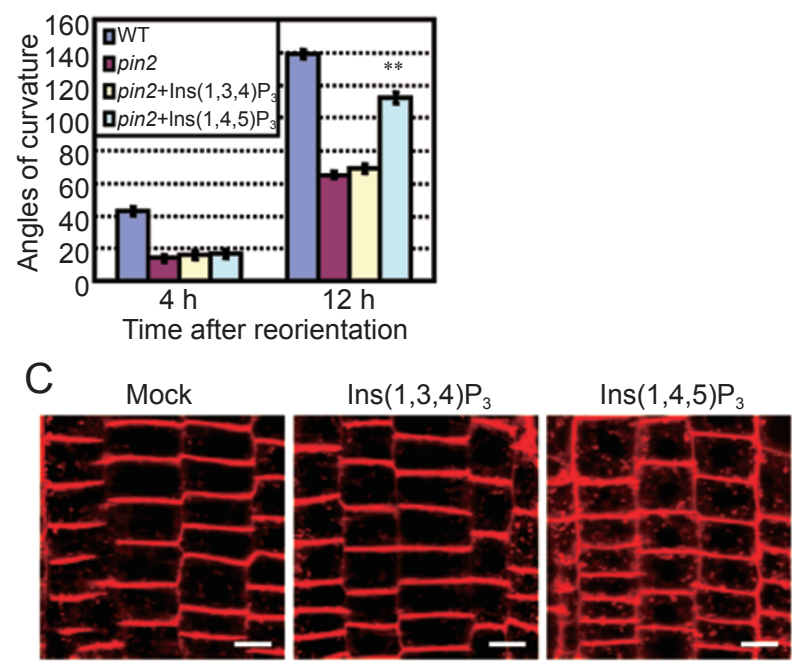

Figure 6 Ins $(1,4,5) \mathrm{P}_{3}$ enhanced root gravitropic response and vesicle trafficking. (A) Root gravitropic responses were promoted by exogenous Ins $(1,4,5) \mathrm{P}_{3}$ under either $90^{\circ}$ (left panel) or $180^{\circ}$ (right panel) reorientations. After treatment with $\operatorname{Ins}(1,4,5) \mathrm{P}_{3}$ or a control solution for $2 \mathrm{~h}$, 3-day-old seedlings were reoriented at $90^{\circ}$ or $180^{\circ}$, and root bending curvatures were measured at intervals of $2 \mathrm{~h}$. Ins $(1,3,4) \mathrm{P}_{3}$ (Inositol 1,3,4-trisphosphate) was used as a control, same in B and C. SAS statistical software was used for statistical analyses $(* P<0.05 ; * * P<0.01)$. Error bars indicate $S E(n=60)$. (B) Exogenous Ins $(1,4,5) P_{3}$ could partially rescue the gravitropic response of the pin2 mutant. Ins $(1,4,5) \mathrm{P}_{3}$ treatment was performed as described in A. Root curvatures were measured after $180^{\circ}$ gravistimulation for 4 or 12 h. Error bars indicate SE $(n=60)$. Statistical analysis using SAS statistical software indicated a significance difference $(* * P<0.01)$ between pin2 and pin2 seedlings treated with $\operatorname{Ins}(1,4,5) P_{3}$. (C) Exogenous Ins $(1,4,5) \mathrm{P}_{3}$-stimulated vesicle trafficking in WT seedlings. After treatment with $\operatorname{Ins}(1,3,4) \mathrm{P}_{3}, \operatorname{Ins}(1,4,5) \mathrm{P}_{3}$, or a control solution, seedlings $(n=20)$ were stained with FM4-64 before observation. Bar $=10 \mu \mathrm{m}$.

indicate a potential relationship between vesicular trafficking and the root gravitropic response and suggest an important role of vesicle trafficking in root gravitropism. There is no significant alteration in vesicle trafficking after either short-term (within $20 \mathrm{~min}$ ) or long-term (1 
or $2 \mathrm{~h}$ ) gravistimulation, which may be because the presumed gravity-induced alteration in vesicular trafficking is transient or completed in a short period that cannot be captured accurately. Improved observation techniques and methods of investigation may lead to more detailed insights.

Because the gravitropic response of the 5 pt $13 \mathrm{mu}-$ tant was not dramatically enhanced, potential roles for other Arabidopsis 5PTases cannot be excluded. Studies employing mutations in multiple 5PTases may reveal a more dramatic influence on root gravitropism and help to address this issue.

5PTase13 affects vesicle trafficking and auxin redistribution under gravistimulation

The altered vesicle trafficking and suppressed responses to the vesicle-trafficking inhibitor BFA under 5 PTase 13 deficiency indicate a role for 5PTase 13 in vesicle trafficking, which has been proposed in animals (for example, in C. elegans and Drosophila, defects in 5PTases including synaptojanin or its homologs result in alterations in synaptic vesicle transport) [47]. Exogenous Ins $(1,4,5) \mathrm{P}_{3}$ enhanced the internalization of FM4-64 in WT, consistent with the increased $\operatorname{Ins}(1,4,5) \mathrm{P}_{3}$ level, and altered vesicle trafficking in the $5 p t 13$ mutant (Figure $6 \mathrm{C}$ ), indicating that the $5 \mathrm{P}$ Tase 13 protein or its substrate $\operatorname{Ins}(1,4,5) \mathrm{P}_{3}$ may exert an essential influence in vesicle transport. The altered cycling of both PIN2 and PIN1 further indicates that 5PTase 13 may influence intracellular vesicle trafficking in a general way. Based on the fact that the intracellular trafficking events in 5 pt13 mutant showed less sensitivity to BFA, the possibility of missorting into less BFA-sensitive sorting pathways might be a potential interpretation.

The PIN2 expression domain was greatly expanded from the root meristem zone to the elongation zone in 5 pt 13 mutant seedlings, consistent with the results of application of exogenous Ins $(1,4,5) \mathrm{P}_{3}$ to WT seedlings. These findings suggest that modified PIN2 expression under 5PTase 13 deficiency may be a result of accumulation of intrinsic substrates such as $\operatorname{Ins}(1,4,5) \mathrm{P}_{3}$. Because PIN2 expression has a direct relationship with the root gravitropic response, the broader PIN2 expression may contribute greatly to the stimulated gravitropic response in the $5 p t 13$ mutant. PIN2 expression was not affected at the transcriptional level either in 5pt13 mutant roots or under Ins $(1,4,5) \mathrm{P}_{3}$ treatment (Figure $2 \mathrm{C}$ ), suggesting a potential mechanism of post-transcriptional regulation. Recently, a dark-induced assay was used to visualize PIN2 turnover in lytic vacuoles [27] and our examination revealed the slower turnover of PIN2 proteins in 5 pt13 mutant (Figure 7).

As the vacuolar targeting of PIN2 was performed in a retromer-dependent way [27], the altered PIN2 sorting under 5PTase13 deficiency was suggested to contribute to the slower PIN2 turnover in lytic vacuoles. Taken together, these results indicate that $5 \mathrm{PTase} 13$ or $\operatorname{Ins}(1,4,5) \mathrm{P}_{3}$ might have a regulatory role in protein turnover via affecting vesicle trafficking-dependent protein sorting, a possibility that is under further investigation.

Together with the fact that PAT and the consequent redistribution of endogenous auxin are essential for grav-

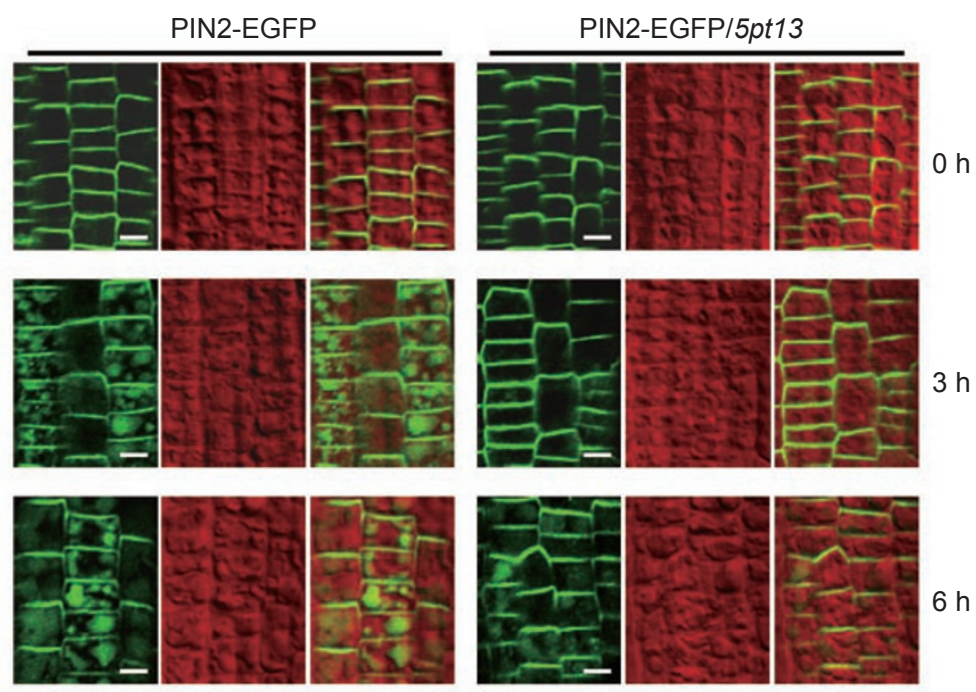

Figure 7 Suppressed dark-induced PIN2 trafficking to lytic vacuoles under 5PTase13 deficiency. Observation on the appearance of PIN2 protein in lytic vacuoles of PIN2-EGFP (leaf panel) or PIN2-EGFP/5pt13 seedlings (right panel). The roots were incubated in darkness for 3 or $6 \mathrm{~h}$ and root epidermal cells were observed. 
ity response of Arabidopsis roots [19, 48], it is suggested that broader PIN2 expression and altered cycling of PIN proteins by modified vesicle trafficking may stimulate gravity-induced auxin redistribution and, consequently, the root gravitropic response. Considering the altered PIN1 cycling, PIN1 may also be involved in the changes in auxin redistribution during the gravitropic response.

In addition, reduced intrinsic Ins $(1,4,5) \mathrm{P}_{3}$ levels caused impaired plant tropistic responses, accompanied by decreased basipetal IAA transport [33], which suggests that Ins $(1,4,5) \mathrm{P}_{3}$ may have a direct or indirect influence on the polar and lateral transport of auxin and may partially account for auxin redistribution under gravistimulation.

\section{Materials and Methods}

\section{Chemical reagents \\ NPA (N0926) was purchased from Duchefa Biochemie (Haar-} lem, The Netherlands). D-myo-inositol 1,4,5-trisphosphate (sodium) (Ins(1,4,5) $\left.\left.\mathrm{P}_{3}, \mathrm{Q}-0145\right)\right)$ and D-myo-inositol 1,3,4-trisphosphate (sodium) $\left(\operatorname{Ins}(1,3,4) \mathrm{P}_{3}, \mathrm{Q}-0135\right)$ were obtained from Echelon (CA, USA). CHX (94271) was purchased from Sangon (Shanghai, China). FM4-64 (T13320) and Shuttle PIP ${ }^{\mathrm{TM}}$ Carrier-1, histone H1 (S-23731) were purchased from Molecular Probes (OR, USA). BFA (B-7651) was purchased from Sigma-Aldrich (MO, USA).

\section{Plant materials and growth conditions}

The Arabidopsis Columbia ecotype was used in the study. Seeds were surface sterilized with $20 \%$ bleach, washed four times with sterile water, and then sown on Murashige and Skoog (MS) medium [49]. Plates were stored for stratification at $4{ }^{\circ} \mathrm{C}$ in darkness for $48-72 \mathrm{~h}$ and then transferred to a phytotron at $22{ }^{\circ} \mathrm{C}$ with a $16 \mathrm{~h} \mathrm{light} / 8 \mathrm{~h}$ dark cycle. Seedlings were vertically grown for the following experiments.

The 5 pt 13 mutant, p35S:5PTase 13 in 5 pt 13 lines, and p35S: 5 PTase 13 transgenic lines used in this study are from a previous study [40]. The 5PTase13 full-length cDNA driven by the CaMV35S promoter was transformed into WT or the 5 pt13 mutant, respectively.

For NPA treatment, seeds were sown on MS medium with gradients of NPA $(0.1,0.5,1$, and $2 \mu \mathrm{M})$, and 8-day-old seedlings vertically grown in light were used for measurement of primary root lengths. For BFA treatment, 6-day-old seedlings were transferred onto medium supplemented with different concentrations of BFA $(2,5$, and $10 \mu \mathrm{M})$ and grown vertically for another 6 days. Then the lengths of the primary roots were measured and calculated. For hypocotyl length measurement, seeds were sown on medium supplemented with 5 or $10 \mu \mathrm{M}$ BFA, and the lengths of hypocotyls of 8-day-old seedlings were measured and calculated.

For all experiments, the average data from three independent experiments are presented, and 50-60 seedlings/line were analyzed in each experiment (as is the case for other measurements in this study). Statistical analysis was performed using SAS statistical software (SAS version 8.0), including the test for homogeneity of variance and 2-tailed $t$-test analysis (as is the case for other measurements in this study).

\section{Root gravitropism assay}

For root gravitropic bending measurement, seeds were germinated as described above and grown on vertically oriented plates for 2 days in the light and 1 days in the dark. All 3-day-old seedlings were transferred to new MS plates before reorientation treatment. In details, seedlings were handled slightly with forceps on hypocotyl tissues, ensuring the root tissues slide gently on the surface so that all the root tips were vertically downward. Then seedlings were incubated in the dark for $1 \mathrm{~h}$ for recovery and adaptation. These manipulations will ensure the uniform orientation of all roots before reorientation treatment, and can avoid mechanical damage to the root tissues. Plates were then rotated by $180^{\circ}$ in darkness, and images were photographed at intervals of $2 \mathrm{~h}$ with a Nikon Coolpix 4500 digital camera. The angles of root curvature were measured at $4,6,8,10,12$, or $24 \mathrm{~h}$ after reorientation with the aid of the image analysis program Image $J$ (version 1.34 for Windows; http://rsb.info.nih.gov/ij/).

For DR5 and DR5/5pt13 seedlings, prior to or after reorientation $\left(90^{\circ}\right.$ or $180^{\circ}$, different times), roots were subjected to GUS staining [50] for $4 \mathrm{~h}$. After being cleared, seedlings were photographed with an Interference Discrepancy Contrast Microscope system (Leica). Two independent lines were analyzed.

Treatment with exogenous Ins $(1,4,5) P_{3}$ or Ins $(1,3,4) P_{3}$

An intracellular delivery system was used to translocate inositol trisphosphates through the cell membrane and into the cytoplasm, and Shuttle PIPTM Carrier-1, histone H1 (Molecular Probes, http://probes.invitrogen.com) was employed to help the $\mathrm{InsP}_{3}$ molecules penetrating through the cell membranes. The delivery system has been proven to be efficient for translocation of biologically active $\mathrm{InsP}_{3}$ into mammalian cells and $\mathrm{PIP}_{2}$ into Arabidopsis root cells [51, 52]. Shuttle PIP Carrier-1 and inositol trisphosphates were mixed immediately prior to the experiments, allowed for equilibration for $5 \mathrm{~min}$, and then added to liquid 1/2 MS medium to generate $10 \mu \mathrm{M} \operatorname{Ins}(1,4,5) \mathrm{P}_{3}$ or $\operatorname{Ins}(1,3,4) \mathrm{P}_{3}$ (inositol $1,3,4$-trisphosphate). A control solution was prepared from an equivalent amount of Shuttle Carrier-1 without inositol trisphosphates in liquid medium. Seedlings were incubated in the above solutions for $2 \mathrm{~h}$, followed by rinse in liquid medium thrice. For root gravitropic responses, 3-day-old vertically grown WT seedlings pretreated with inositol trisphosphates were slightly replaced onto the MS plates and incubated in the dark for $1 \mathrm{~h}$ for recovery and adaptation. Then seedlings were subjected to $90^{\circ}$ or $180^{\circ}$ reorientations in the dark, and bending curvatures were measured in intervals of $2 \mathrm{~h}$.

For examining the vesicle trafficking, 4-day-old WT seedlings were stained with FM4-64 following treatment with inositol trisphosphates as described above (details are described in "Observation of vesicle trafficking under FM4-64 staining and BFA treatment"). For observation of PIN2 expression, after treatment with $\operatorname{Ins}(1,4,5) \mathrm{P}_{3}, \operatorname{Ins}(1,3,4) \mathrm{P}_{3}$ or control solution in the absence or presence of $50 \mu \mathrm{M}$ CHX, PIN2-EGFP seedlings were observed and photographed with a confocal laser scanning microscope. PIN2p::GUS seedlings were subjected to GUS staining with $1 / 10$ fold X-Gluc concentration, and photographed with an Interference Discrepancy Contrast Microscope system (Leica) after being cleared.

\section{Measurement of Ins $(1,4,5) P_{3}$ content}

Seedlings (4-day old) treated with exogenous $\operatorname{Ins}(1,4,5) \mathrm{P}_{3}$ or 
control solution as described above were used for measuring the exogenous Ins $(1,4,5) \mathrm{P}_{3}$ contents. A total of $300 \mathrm{mg}$ of freshly ground tissue was used for extracting $\operatorname{Ins}(1,4,5) \mathrm{P}_{3}$ according to the previous report by Burnette et al. [12]. Intrinsic Ins $(1,4,5) \mathrm{P}_{3}$ content was measured using the D-myo-inositol-1,4,5-trisphosphate $\left[{ }^{3} \mathrm{H}\right]$ assay kit (Amersham-Pharmacia Biotech). The $\operatorname{Ins}(1,4,5) \mathrm{P}_{3}$ content in the samples was interpolated from a standard curve generated with known amounts of $\operatorname{Ins}(1,4,5) \mathrm{P}_{3}$. Assays were performed in triplicate, and the experiment was repeated twice.

\section{Genetic crosses}

DR5-GUS, PIN2-EGFP, and PIN1-EYFP marker lines were kindly provided by Dr Jian Xu (Utrecht University, The Netherlands), and genetic crosses were carried out by removing the petals, sepals, and androecia from large green buds, followed by artificial fecundation on the next day. The DR5-GUS, PIN2EGFP, and PIN1-EYFP cassette were individually transferred into the 5 pt 13 mutants. The homozygous offspring DR5/5pt13, PIN2EGFP/5pt13, and PIN1-EYFP/5pt13 were used for the following experiments.

\section{Quantitative real-time RT-PCR analysis}

Total RNA was isolated from root tissue of 4-day-old WT or $5 p t 13$ mutant seedlings using Trizol reagent (Invitrogen) and then reverse-transcribed according to the manufacturer's instructions (ReverTra Ace kit, TOYOBO). Quantitative real-time RT-PCR analysis was performed with the RotorGene 3000 system (Corbett Research) using the SYBR green detection protocol (Realtime PCR Master Mix, TOYOBO). The amounts of transcripts were calculated using the comparative threshold cycle method. Differential gene expression is indicated as relative expression levels (percentage) compared with the internal control ACTIN7. The primers used were as follows: PIN2 (5'-CCG TGG GGC TAA GCT TCT CAT CT-3' and 5'-AGC TTT CCG TCG TCT CCT ATC TCC-3'), ACTIN7 (5'-TTC CCG TTC TGC GGT AGT GG-3' and 5'-CCG GTA TTG TGC TCG ATT CTG-3').

Observation of vesicle trafficking with FM4-64 staining and BFA treatment

Roots of WT, 5pt13, and 5PTase13-overexpressing seedlings were stained with FM4-64 $(5 \mu \mathrm{M})$ for $10 \mathrm{~min}$, followed by two rinses. After incubation for $40 \mathrm{~min}$ at room temperature, red fluorescence was observed with a confocal laser scanning microscope (FITC488, Zeiss LSM500). FM4-64 fluorescence was excited with a 543-nm argon ion laser and a 600-nm long-pass emission filter. Images within a single experiment were captured with the same imaging system parameters.

For BFA treatment, seedlings were co-treated with BFA $(100 \mu \mathrm{M})$ and FM4-64 $(5 \mu \mathrm{M})$ for $30 \mathrm{~min}$ before observation. Each experiment above was repeated thrice.

Quantitative analyses of vesicles were performed using the corresponding confocal images. For an individual seedling, the vesicle number was calculated from the average of 10 independent root cells, and 30 seedlings of each line were measured for each treatment.

Visualization of PIN2 localization and cycling of PIN proteins

Roots of PIN2-EGFP and PIN2-EGFP/5pt13 plants were ob- served and imaged with a confocal laser scanning microscope at a wavelength of $488 \mathrm{~nm}$ (EGFP), and the lengths of the PIN2 expression regions were measured with the Zeiss LSM Image Browser program. For dark-induced PIN2 trafficking to vacuoles, root was incubated in darkness and root epidermal cells were observed. The experiments were repeated thrice.

To visualize the cycling of PIN1 or PIN2, seedlings were treated with BFA $(50 \mu \mathrm{M})$ for the indicated time before observation. To recover the polar localization of the PIN1 or PIN2 proteins, BFA washout was conducted following a 2-h treatment with BFA $(50 \mu \mathrm{M})$. Visualization of PIN1 was performed under a wavelength of 514 nm (EYFP).

For quantitative analyses of PINs cycling, percentage of root cells containing BFA compartments after BFA treatment were calculated with the help of corresponding confocal microscope images. After BFA washout, percentage of root cells with PIN2-containing vesicles or those still containing BFA compartments were calculated. In total, 30 individual seedlings were analyzed for each treatment. The total fluorescent intensity and size of BFA compartments in each cell (before washout of BFA) was measured using laser confocal microscope LSM 510 software (ver 3.2), based on the confocal images photographed with the same parameters. More than 100 cells were analyzed for each line.

\section{Acknowledgments}

This study was supported by the Chinese Academy of Sciences (KSCX2-YW-N-016) and the National Natural Science Foundation of China (Grants 30721061 and 30740006).

\section{References}

1 Stevenson JM, Perera IY, Heilmann I, et al. Inositol signaling and plant growth. Trends Plant Sci 2000; 5:252-258.

2 Meijer HJ, Munnik T. Phospholipid-based signaling in plants. Annu Rev Plant Biol 2003; 54:265-306.

3 Xue HW, Chen X, Li G. Involvement of phospholipid signaling in plant growth and hormone effects. Curr Opin Plant Biol 2007; 10:483-489.

4 Berdy SE, Kudla J, Gruissem W, Gillaspy GE. Molecular characterization of At5PTase1, an inositol phosphatase capable of terminating inositol trisphosphate signaling. Plant Physiol 2001; 126:801-810.

5 Ercetin ME, Gillaspy GE. Molecular characterization of an Arabidopsis gene encoding a phospholipid-specific inositol polyphosphate 5-phosphatase. Plant Physiol 2004; 135:938946.

6 Zhong R, Burk DH, Morrison WH, Ye ZH. FRAGILE FIBER3, an Arabidopsis gene encoding a type II inositol polyphosphate 5-phosphatase, is required for secondary wall synthesis and actin organization in fiber cells. Plant Cell 2004; 16:3242-3259.

7 Zhong R, Ye ZH. Molecular and biochemical characterization of three WD-repeat-domain-containing inositol polyphosphate 5-phosphatases in Arabidopsis thaliana. Plant Cell Physiol 2004; 45:1720-1728.

8 Carland FM, Nelson T. COTYLEDON VASCULAR PATTERN2-mediated inositol $(1,4,5)$ trisphosphate signal transduction is essential for closed venation patterns of Arabidop- 
sis foliar organs. Plant Cell 2004; 16:1263-1275.

9 Lin WH, Wang Y, Mueller-Roeber B, et al. At5PTase13 modulates cotyledon vein development through regulating auxin homeostasis. Plant Physiol 2005; 139:1677-1691.

10 Gunesekera B, Torabinejad J, Robinson J, Gillaspy GE. Inositol polyphosphate 5-phosphatases 1 and 2 are required for regulating seedling growth. Plant Physiol 2007; 143:14081417.

11 Sanchez JP, Chua NH. Arabidopsis PLC1 is required for secondary responses to abscisic acid signals. Plant Cell 2001; 13:1143-1154.

12 Burnette RN, Gunesekera BM, Gillaspy GE. An Arabidopsis inositol 5-phosphatase gain-of-function alters abscisic acid signaling. Plant Physiol 2003; 132:1011-1019.

13 Hangarter RP. Gravity, light and plant form. Plant Cell Environ 1997; 20:796-800.

14 Tasaka M, Kato T, Fukaki H. The endodermis and shoot gravitropism. Trends Plant Sci 1999; 4:103-107.

15 Muday GK. Auxins and tropisms. J Plant Growth Regul 2001; 20:226-243.

16 Blancaflor EB, Masson PH. Plant gravitropism: unraveling the ups and downs of a complex process. Plant Physiol 2003; 133:1677-1690.

17 Muller A, Guan C, Galweiler L, et al. AtPIN2 defines a locus of Arabidopsis for root gravitropism control. EMBO J 1998; 17:6903-6911.

18 Friml J, Yang X, Michniewicz M, et al. A PINOID-dependent binary switch in apical-basal PIN polar targeting directs auxin efflux. Science 2004; 306:862-865.

19 Rashotte AM, Brady SR, Reed RC, Ante SJ, Muday GK. Basipetal auxin transport is required for gravitropism in roots of Arabidopsis. Plant Physiol 2000; 122:481-490.

20 Li L, Xu J, Xu ZH, Xue HW. Brassinosteroids stimulate plant tropisms through modulation of polar auxin transport in Brassica and Arabidopsis. Plant Cell 2005; 10:2738-2753.

21 Friml J, Wisniewska J, Benkova E, Mendgen K, Palme K. Lateral relocation of auxin efflux regulator PIN3 mediates tropism in Arabidopsis. Nature 2002; 415:806-809.

22 Geldner N, Friml J, Stierhof YD, Jurgens G, Palme K. Auxin transport inhibitors block PIN1 cycling and vesicle trafficking. Nature 2001; 413:425-428.

23 Geldner N, Anders N, Wolters H, et al. The Arabidopsis GNOM ARF-GEF mediates endosomal recycling, auxin transport, and auxin-dependent plant growth. Cell 2003; 112:219230.

24 Dhonukshe P, Aniento F, Hwang I, et al. Clathrin-mediated constitutive endocytosis of PIN auxin efflux carriers in Arabidopsis. Curr Biol 2007; 17:520-527.

25 Kleine-Vehn J, Dhonukshe P, Sauer M, et al. ARF GEF-dependent transcytosis and polar delivery of PIN auxin carriers in Arabidopsis. Curr Biol 2008; 18:526-531.

26 Jaillais Y, Fobis-Loisy I, Miege C, Rollin C, Gaude T. AtSNX1 defines an endosome for auxin-carrier trafficking in Arabidopsis. Nature 2006; 443:106-109.

27 Kleine-Vehn J, Leitner J, Zwiewka M, et al. Differential degradation of PIN2 auxin efflux carrier by retromer-dependent vacuolar targeting. Proc Natl Acad Sci USA 2008; 105:1781217817.

28 Freyberg Z, Siddhanta A, Shields D. "Slip, sliding away": phospholipase D and the Golgi apparatus. Trends Cell Biol 2003; 13:540-546.

29 Jenkins GM, Frohman MA. Phospholipase D: a lipid centric review. Cell Mol Life Sci 2005; 62:2305-2316.

30 Monteiro D, Liu Q, Lisboa S, Scherer GE, Quader H, Malho R. Phosphoinositides and phosphatidic acid regulate pollen tube growth and reorientation through modulation of $\left[\mathrm{Ca}^{2+}\right]_{\mathrm{cyt}}$ and membrane secretion. J Exp Bot 2005; 56:1665-1674.

31 Li G, Xue HW. Arabidopsis PLDל22 regulates vesicle trafficking and is required for auxin response. Plant Cell 2007; 19:281-295.

32 Perera IY, Heilmann I, Boss WF. Transient and sustained increases in inositol 1,4,5-trisphosphate precede the differential growth response in gravistimulated maize pulvini. Proc Natl Acad Sci USA 1999; 96:5838-5843.

33 Perera IY, Heilmann I, Chang SC, Boss WF, Kaufman PB. A role for inositol 1,4,5-trisphosphate in gravitropic signaling and the retention of cold-perceived gravistimulation of oat shoot pulvini. Plant Physiol 2001; 125:1499-1507.

34 Perera IY, Hung CY, Brady S, Muday GK, Boss WF. A universal role for inositol 1,4,5-trisphosphate-mediated signaling in plant gravitropism. Plant Physiol 2006; 140:746-760.

$35 \mathrm{Xu}$ J, Scheres B. Dissection of Arabidopsis ADPRIBOSYLATION FACTOR1 function in epidermal cell polarity. Plant Cell 2005; 17:1-12.

36 Bolte S, Talbot C, Boutte Y, Catrice O, Read ND, SatiatJeunemaitre B. FM-dyes as experimental probes for dissecting vesicle trafficking in living plant cells. J Microsc 2004; 214:159-173.

37 Nebenfuhr A, Ritzenthaler C, Robinson DG. Brefeldin A: deciphering an enigmatic inhibitor of secretion. Plant Physiol 2002; 130:1102-1108.

38 Grebe M, Xu J, Mobius W, et al. Arabidopsis sterol endocytosis involves actin-mediated trafficking via ARA6-positive early endosomes. Curr Biol 2003; 13:1378-1387.

39 Ulmasov T, Murfett J, Hagen G, Guilfoyle TJ. Aux/ IAA proteins repress expression of reporter genes containing natural and highly active synthetic auxin response elements. Plant Cell 1997; 9:1963-1971.

40 Buer CS, Muday GK. The transparent testa4 mutation prevents flavonoid synthesis and alters auxin transport and the response of Arabidopsis roots to gravity and light. Plant Cell 2004; 16:1191-1205.

41 Chen X, Lin WH, Wang Y, Luan S, Xue HW. An inositol polyphosphate 5-phosphatase functions in PHOTOTROPIN1 signaling in Arabidopsis by altering cytosolic $\mathrm{Ca}^{2+}$. Plant Cell 2008; 20:353-366.

42 Plieth C, Trewavas AJ. Reorientation of seedlings in the earth's gravitational field induces cytosolic calcium transients. Plant Physiol 2002; 129:786-796.

43 Kato T, Morita MT, Fukaki H, et al. SGR2, a phospholipaselike protein, and ZIG/SGR4, a SNARE, are involved in the shoot gravitropism of Arabidopsis. Plant Cell 2002; 14:33-46.

44 Yano D, Sato M, Saito C, Sato MH, Morita MT, Tasaka M. A SNARE complex containing SGR3/AtVAM3 and ZIG/VTI11 in gravity-sensing cells is important for Arabidopsis shoot gravitropism. Proc Natl Acad Sci USA 2003; 100:8589-8594.

45 Zhang Y, Grant B, Hirsh D. RME-8, a conserved J-domain protein, is required for endocytosis in Caenorhabditis elegans. 
Mol Biol Cell 2001; 12:2011-2021.

46 Chang HC, Hull M, Mellman I. The J-domain protein Rme-8 interacts with Hsc70 to control clathrin-dependent endocytosis in Drosophila. J Cell Biol 2004; 164:1055-1064.

47 Song W, Zinsmaier KE. Endophilin and synaptojanin hook up to promote synaptic vesicle endocytosis. Neuron 2003; 40:665-667.

48 Rashotte AM, DeLong A, Muday GK. Genetic and chemical reductions in protein phosphatase activity alter auxin transport, gravity response, and lateral root growth. Plant Cell 2001; 13:1683-1697.

49 Murashige T, Skoog F. A revised medium for rapid growth and bioassays with tobacco tissue cultures. Physiol Plant 1962; 15:473-497.

50 Jefferson RA, Kavanagh TA, Bevan MW. GUS fusions: $\beta$-Glucuronidase as a sensitive and versatile gene fusion marker in higher plants. EMBOJ 1987; 6:3901-3907.

51 Ozaki S, DeWald DB, Shope JC, Chen J, Prestwich GD. Intracellular delivery of phosphoinositides and inositol phosphates using polyamine carriers. Proc Natl Acad Sci USA 2000; 97:11286-11291.

52 Lee Y, Kim YW, Jeon BW, et al. Phosphatidylinositol 4,5 -bisphosphate is important for stomatal opening. Plant $J$ 2007; 52:803-816.

(Supplementary information is linked to the online version of the paper on the Cell Research website.) 